\title{
On the attribution of contributions of atmospheric trace gases to emissions in atmospheric model applications
}

\author{
V. Grewe ${ }^{1}$, E. Tsati ${ }^{1}$, and P. Hoor ${ }^{2}$ \\ ${ }^{1}$ Deutsches Zentrum für Luft- und Raumfahrt, Institut für Physik der Atmosphäre, Oberpfaffenhofen, Germany \\ ${ }^{2}$ Institut für Physik der Atmosphäre, Universität Mainz, Mainz, Germany
}

Received: 11 May 2010 - Published in Geosci. Model Dev. Discuss.: 10 June 2010

Revised: 9 August 2010 - Accepted: 16 August 2010 - Published: 12 October 2010

\begin{abstract}
We present an improved tagging method, which describes the combined effect of emissions of various species from individual emission categories, e.g. the impact of both, nitrogen oxides and non-methane hydrocarbon emissions on ozone. This method is applied to two simplified chemistry schemes, which represent the main characteristics of atmospheric ozone chemistry. Analytical solutions are presented for this tagging approach. In the past, besides tagging approaches, sensitivity methods were used, which estimate the contributions from individual sources based on differences in two simulations, a base case and a simulation with a perturbation in the respective emission category. We apply both methods to our simplified chemical systems and demonstrate that potentially large errors (factor of 2) occur with the sensitivity method, which depend on the degree of linearity of the chemical system. This error depends on two factors, the ability to linearise the chemical system around a base case, and second the completeness of the contributions, which means that all contributions should principally add up to $100 \%$. For some chemical regimes the first error can be minimised by employing only small perturbations of the respective emission, e.g. 5\%. The second factor depends on the chemical regime and cannot be minimized by a specific experimental set-up. It is inherent to the sensitivity method. Since a complete tagging algorithm for global chemistry models is difficult to achieve, we present two error metrics, which can be applied for sensitivity methods in order to estimate the potential error of this approach for a specific application.
\end{abstract}

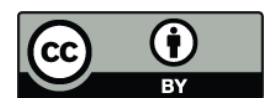

Correspondence to: V. Grewe (volker.grewe@dlr.de)

\section{Introduction}

The attribution of climate change to changes in emission of greenhouse gases and precursors has been an issue of serious concern over several decades. Recently, EU-projects like QUANTIFY and ATTICA aimed at identifying the impact of transport sectors on climate (Fuglestvedt et al., 2009; Hoor et al., 2009; Lee et al., 2010). Wang et al. (2009) pointed out that there is a difference in the nature of the two key topics (1) attribution of climate change to sectoral emissions and (2) evaluation of emission control scenarios, which also requires different methodologies. The attribution of concentrations to emissions is important to attribute climate change, which depends on absolute contributions, to sectoral emissions. In contrast, emission control scenarios for attaining air quality or climate change goals require knowledge on the sensitivity of atmospheric concentrations toemissions. It is important to acknowledge that these two topics might differ greatly.

Figure 1 sketches briefly the idea of either method. The general settings are given in Fig. 1a, which shows an arbitrary relation between emissions of $\mathrm{NO}_{\mathrm{x}}$ and the response in ozone. Two simulations, a base case and a perturbation simulation, where an emission category is changed by the factor $\alpha$, are indicated. The line through both simulation points (green) is an approximation of the tangent (dashed line). Basically (more details are given in Sect. 3), the sensitivity method uses the tangent approximation, whereas the tagging method is based on the origin line to determine the ratio between the change in ozone mass and the emission of $\mathrm{NO}_{\mathrm{x}}$.

Obviously, for species, which are controlled by linear processes, like ${ }^{222} \mathrm{Rn}$ or $\mathrm{SF}_{6}$, both approaches will lead to identical results (Fig. 1b). For non-linear systems both methods

Published by Copernicus Publications on behalf of the European Geosciences Union. 

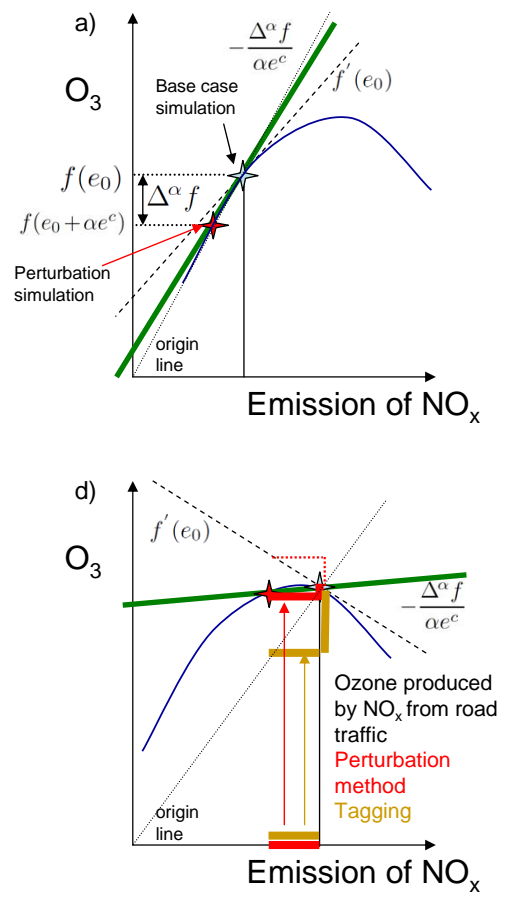
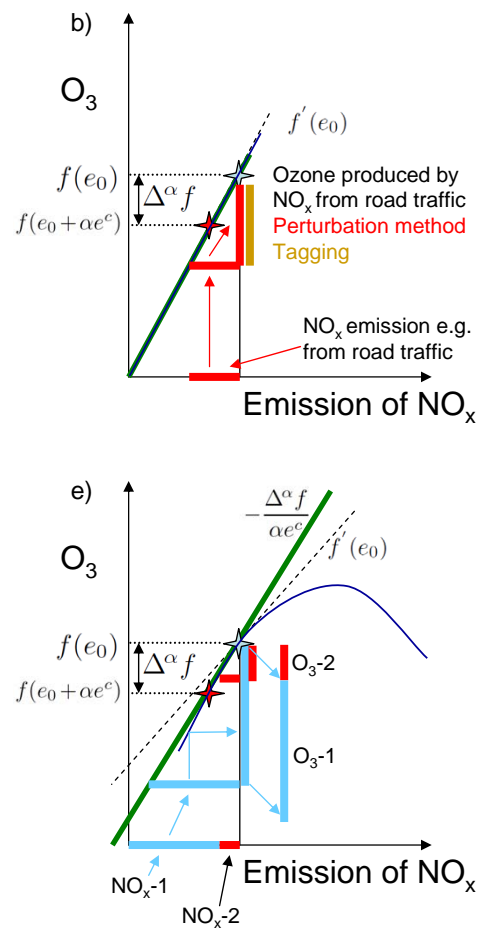
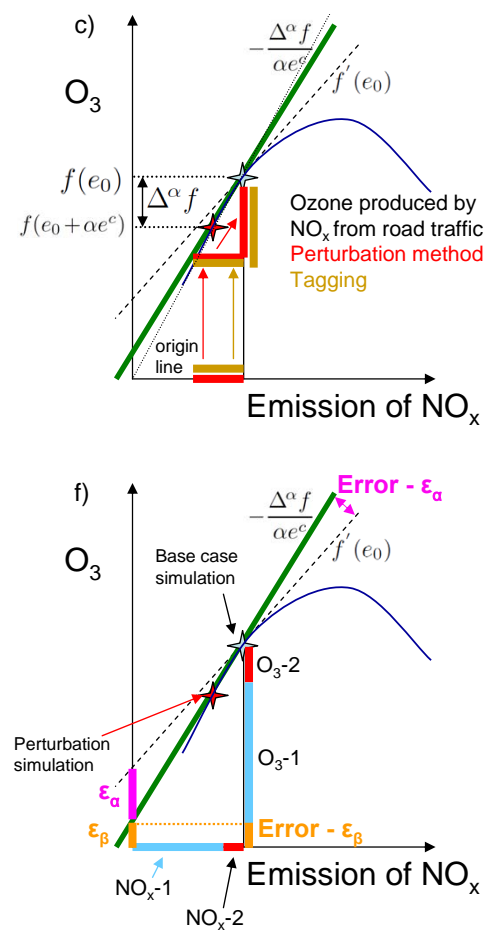

Fig. 1. Illustration of the sensitivity method (pair of simulations) to derive contributions from emission categories and intercomparison with the tagging method. The ozone concentration in arbitrary units is shown as a function of the emission of $\mathrm{NO}_{\mathrm{x}}$. Two simulations (base case and a simulation in which the emissions $e^{\mathrm{c}}$ is changed by a factor $\alpha$ ) are indicated with stars. The derivative is added as a tangent for the base case (dashed line). The line through the base case simulation and the origin (origin line) is dotted. The green line shows the estimated derivative, based on the two simulations. (a) General settings and calculation of the derivative. (b) Assumption of linearity in ozone chemistry for illustration purpose. An arbitrary $\mathrm{NO}_{\mathrm{x}}$ emission (horizontal red line) is considered. The vertical red and brown lines indicate the ozone contributions caused by this $\mathrm{NO}_{\mathrm{x}}$ source (sensitivity method in red and tagging in brown) giving identical results. (c) As (b) but for the assumption of a non-linear ozone chemistry, however in a situation, which is close to the linear case. The green and dotted lines are used to calculate the contributions based on the sensitivity and tagging method, respectively. (d) As (c), but for a situation, which is far from the linear regime. (e) Calculation of the ozone contributions; two emission categories are considered $\left(\mathrm{NO}_{\mathrm{x}}-1\right.$ : light blue, $\mathrm{NO}_{\mathrm{x}}-2$ : red) and the ozone contributions $\mathrm{O}_{3}-1$ and $\mathrm{O}_{3}-2$ indicated with vertical lines. (f) Error analysis; the two errors $\epsilon_{\alpha}$ (magenta) and $\epsilon_{\beta}$ (orange), which describe uncertainties associated with the determination of the tangent and the total estimate of all contributions (intersection of $y$-axis and tangent) (see Sect. 6). Note, the origin line for tagging represents the equality of all emitted $\mathrm{NO}_{\mathrm{x}}$ molecules to take part in a reaction, which implies that a subset of $\mathrm{NO}_{\mathrm{x}}$ molecules, e.g. from the sources category "road traffic", produces a sub-set of ozone molecules in a linear relation-ship (= origin line) for a non-linear chemistry (blue line).

might deviate only little (Fig. 1c), if the approximated tangent and the origin line differ only slightly. However, as soon as the system becomes non-linear, differences between the approaches have the potential to increase largely (Fig. 1d).

Therefore, two aspects are important, the accuracy of the determination of the tangent and the deviation of the tangent from the origin line.

Generally, as pointed out by Wang et al. (2009), it is important to differentiate between the two questions concerning attribution and emission control scenarios and to concede that the answers to these questions require two different methodologies. The attribution of atmospheric concentrations to emissions (and sources in general) in a numerical simulation framework can be obtained by a tagging methodology, whereas developing effective emission control scenarios is also obtained by sensitivity methods.
A large number of methodologies for both approaches exist (see e.g. Wang et al., 2009). The tagging methodologies are differently designed and implemented, but they have in common that additional "tagged species" are included in the models, to which specific emissions are assigned and which undergo the same loss processes as the respective un-tagged species. In addition, also products arising from the respective compounds can be tagged so that the impact on the whole chemical system can be determined. For example, tagging nitrogen oxide emissions from road traffic implies that every species, which contains an " $\mathrm{N}$ " atom is doubled in the chemical system, tagged with a "road traffic" (rt), and all chemical reactions doubled without changing the chemical system, e.g.:

$$
\mathrm{NO}_{2}+\mathrm{OH} \longrightarrow \mathrm{HNO}_{3}
$$


is doubled by

$$
\mathrm{NO}_{2}^{\mathrm{rt}}+\mathrm{OH} \longrightarrow \mathrm{HNO}_{3}^{\mathrm{rt}}+\mathrm{OH} \text {. }
$$

Note that on the left side of Reaction (R2) all untagged reactants (educts) appear as products, which ensures that the tagging diagnostic does not affect the chemistry. This method ensures that a closed budget for all nitrogen emissions can be achieved. In addition, the impact on ozone is included, by calculating the ozone production by road traffic $\mathrm{NO}_{\mathrm{x}}$ via the reactions

$$
\begin{aligned}
\mathrm{NO}+\mathrm{HO}_{2} & \longrightarrow \mathrm{NO}_{2}+\mathrm{OH} \\
\mathrm{NO}^{r t}+\mathrm{HO}_{2} & \longrightarrow \mathrm{NO}_{2}^{\mathrm{rt}}+\mathrm{O}_{3}^{\mathrm{rt}}+\mathrm{HO}_{2} .
\end{aligned}
$$

Since this approach increases the amount of species and chemical reaction drastically, simplifications of the tagging chemistry scheme are applied (e.g. Horowitz and Jacob, 1999; Zimmermann et al., 1999; Grewe, 2004), which map the detailed chemistry scheme onto the main families, e.g. $\mathrm{NO}_{\mathrm{y}}$, and ozone and employ the chemical production and loss terms from the detailed chemical system, which ensures a closed budget and detailed analysis even in multi-decadal climate-chemistry model simulations (Grewe, 2007, 2009). Note, that kinetics of the reactions are not affected by these methods, in contrast to isotope tagging methods (e.g. Gromov et al., 2010), where the rate constants for the tagged species may differ from the untagged respective species. However, in both cases the tagging method is a diagnostic and does not affect the simulated chemistry.

However, to our knowledge, none of the tagging schemes take into account the competing effect of nitrogen oxides and hydrocarbons/volatile organic compounds (VOCs) on ozone production and hence ozone concentration. In this investigation, we propose a tagging methodology, which includes the non-linear impacts of emission categories (e.g. road traffic, biomass burning, etc.). This means that each category may include emissions of different species, like $\mathrm{NO}_{\mathrm{x}}$ and VOCs. To illustrate this tagging methodology, we introduce 2 slightly different, but very simple artificial chemical systems, which, however, represent the main characteristics of atmospheric ozone chemistry. They consist of two different precursors $\mathrm{X}$ and $\mathrm{Y}$, representing VOCs and $\mathrm{NO}_{\mathrm{y}}$ and a species $Z$, which represents ozone.

These chemical systems can be solved analytically, and we further analyse differences between methodologies, which were used in the past to calculate contributions from emission categories, which are based either on sensitivity analysis or tagging methodologies.

The chemical systems are introduced in the next section (Sect. 2). Section 3 describes the two different approaches, which were used in the past to attribute concentrations to emission categories. In Sect. 4 we present the analytical steady state solutions for the chemical systems and the two different attribution methodologies. The accurate contributions are given by the tagging method. The errors arising from the sensitivity method are discussed in Sect. 5. The implications for global model studies and recommendations are given in Sect. 6.

\section{Two simplified atmospheric chemical systems}

In order to investigate the differences in the various methods, which aim at quantifying contributions of emissions to concentrations of atmospheric constituents, we define two simple chemical reaction systems. The two reaction systems differ in the degree of linearity. The reactive components and their reaction system are aiming at representing main characteristics of tropospheric ozone chemistry. Both reaction systems consist of 3 species, $\mathrm{X}, \mathrm{Y}$, and $\mathrm{Z}$, and $\mathrm{X}, \mathrm{Y}$, and $\tilde{\mathrm{Z}}$ and differ only in the formulation of one loss reaction for $Z$ and $\tilde{Z}$, respectively (see below). The three species can be regarded as $\mathrm{HO}_{\mathrm{x}}, \mathrm{NO}_{\mathrm{x}}$ and ozone or more general $\mathrm{VOC}, \mathrm{NO}_{\mathrm{y}}$, and ozone. The species $\mathrm{X}$ and $\mathrm{Y}$ are precursors of $\mathrm{Z}$ and $\tilde{Z}$, respectively. They have emissions $E_{\mathrm{X}}$ and $E_{\mathrm{Y}}$ and an atmospheric loss, which is independent from each other in our idealised approach. Hence, this reflects losses by dry or wet deposition, or a certain atmospheric lifetime. For simplicity reasons we choose constant lifetimes $\tau_{X}$ and $\tau_{Y}$. An overview on the used variables can be found in Table 1 .

The species $\mathrm{Z}$ is characterised by atmospheric chemical production and loss, only. It is produced by a reaction with $\mathrm{X}$ and $\mathrm{Y}$ :

$\mathrm{X}+\mathrm{Y} \longrightarrow \mathrm{Z}+\mathrm{X}+\mathrm{Y}$,

and destroyed by reaction with either $\mathrm{X}$ and $\mathrm{Y}$ :

$\mathrm{X}+\mathrm{Z} \longrightarrow \mathrm{X}$

$\mathrm{Y}+\mathrm{Z} \longrightarrow \mathrm{Y}$.

The second reaction system is very similar and only the loss Reaction (R7) is replaced:

$$
\begin{aligned}
\mathrm{X}+\mathrm{Y} & \longrightarrow \tilde{\mathrm{Z}}+\mathrm{X}+\mathrm{Y} \\
\mathrm{X}+\tilde{\mathrm{Z}} & \longrightarrow \mathrm{X} \\
\mathrm{Y}+\mathrm{Y}+\tilde{\mathrm{Z}} & \longrightarrow \mathrm{Y}+\mathrm{Y} .
\end{aligned}
$$

The reaction rates of Reactions (R5)-(R10) are $P_{X Y}, D_{\mathrm{X}}$, $D_{\mathrm{Y}_{1}}$, and $D_{\mathrm{Y}_{2}}$. Hence these simplified atmospheric chemical systems can be described by

$$
\begin{aligned}
& \dot{X}=E_{\mathrm{X}}-\tau_{\mathrm{X}}^{-1} X \\
& \dot{Y}=E_{\mathrm{Y}}-\tau_{\mathrm{Y}}^{-1} Y \\
& \dot{Z}=P_{\mathrm{XY}} X Y-D_{\mathrm{X}} X Z-D_{\mathrm{Y}_{1}} Y Z \\
& \dot{\tilde{Z}}=P_{\mathrm{XY}} X Y-D_{\mathrm{X}} X \tilde{Z}-D_{\mathrm{Y}_{2}} Y Y \tilde{Z}
\end{aligned}
$$

Reaction (R5) resembles the ozone production by Reaction (R3), since this reaction is the limiting step in the production of ozone by photolysis of $\mathrm{NO}_{2}$ and subsequent reaction of the gained atomic oxygen with molecular oxygen to produce ozone. The loss reactions of $\mathrm{Z}$ and $\tilde{\mathrm{Z}}$ are referring to ozone loss reactions with $\mathrm{OH}$ and $\mathrm{HO}_{2}$, and $\mathrm{NO}$, respectively. 
Table 1. Overview on variables.

\begin{tabular}{|c|c|}
\hline Symbol/Variable & Description \\
\hline $\begin{array}{l}X, Y, Z, \tilde{Z} \\
\text { non-tilded } \\
\text { tilded variables }\end{array}$ & $\begin{array}{l}\text { Reactants of the chemical system; can be regarded as VOCs, } \mathrm{NO}_{\mathrm{y}} \text { and } \mathrm{O}_{3} \\
\text { first, more linear chemical system: no tilde } \\
\text { second, non-linear chemical system: with tilde for all quantities which differ from the first system }\end{array}$ \\
\hline \multicolumn{2}{|c|}{ In the following all tilded variables are omitted. } \\
\hline$E_{X}, E_{Y}$ & total emission of $X$ and $Y$ \\
\hline$\tau_{X}, \tau_{Y}$ & Lifetime of $X$ and $Y$ \\
\hline$E_{X, i}, E_{Y, i}$ & Emission of $X$ and $Y$ of emission category $i$ \\
\hline$X_{i}, Y_{i}, Z_{i}$ & Tagged species with respect to emission category $i$ \\
\hline$f$ & Ozone as a function of total emissions \\
\hline$\delta^{\alpha} f$ & Contribution of emission category $i$ to the concentration of $f$ \\
\hline$\delta^{\alpha} Z$ & Contribution of emission category $i$ to the concentration of $Z$ \\
\hline$X^{\mathrm{eq}}, Y^{\mathrm{eq}}, Z^{\mathrm{eq}}$ & Equilibrium solutions \\
\hline$E$ & $\begin{array}{l}\text { Relative error in the calculation of the contribution of emission category } i \text { to the concentration of } Z \\
\text { wrt. the tagging method }\end{array}$ \\
\hline$\epsilon_{\alpha}$ & Error in the determination of the tangent (see Fig. 1f) \\
\hline$\epsilon_{\beta}$ & $\begin{array}{l}\text { Error in the determination of completeness of the contribution calculations, } \\
\text { i.e. to which content all contributions add up to } 100 \% \text { of } Z\end{array}$ \\
\hline
\end{tabular}

\section{Methodologies}

The main focus of our investigation is the calculation of contributions from individual sources to the concentration of a specific trace gas. One could generally ask whether there is a solution to this problem at all, or whether there is a unique answer to it. Since it is generally believed that, e.g., air traffic emissions contribute to the atmospheric ozone burden with a well defined ozone amount, this motivates a positive answer to both questions. This reduces the question to how this contribution can be quantified.

In the following, we will concentrate on two ways the contribution has been quantified in the past. First, the tagging method (Sect. 3.2), which represents the true contributions, since it is simply calculated by following the reaction pathways. Secondly, the sensitivity approach (Sect. 3.3), with which contributions are calculated by reducing the target emission by a given fraction. We are discussing the methodologies in the framework of the simplified atmospheric chemical systems, described in Sect. 2. An overview on the used variables can be found in Table 1 .

\subsection{Emission sectors}

All emissions can be described by a number of sectors (here $=n$, e.g. road traffic, biomass burning, etc.), which we denote with $i=1, \ldots, n$. Each sector has emissions of primary gases. In our example we denote them with $E_{\mathrm{X}, i}$ and $E_{\mathrm{Y}, i}$, with

$\sum_{i=1}^{n} E_{\mathrm{X}, i}=E_{\mathrm{X}}$ and

$$
\sum_{i=1}^{n} E_{\mathrm{Y}, i}=E_{\mathrm{Y}}
$$

\subsection{Contributions following reaction pathways (tagging method)}

In this section, we define contributions of individual sectors to the concentration of individual species by analysing the reaction pathways. Each species is decomposed into $n$ subspecies, which define the concentration, by which an individual sector contributes to the regarded species.

With respect to our chemical systems, we have then the sub-species $\mathrm{X}_{i}, \mathrm{Y}_{i}, \mathrm{Z}_{i}$, and $\tilde{\mathrm{Z}}_{i}$. Their concentrations $X_{i}, Y_{i}$, $Z_{i}$, and $\tilde{Z}_{i}$ are those parts of the concentrations $X, Y, Z$, and $\tilde{Z}$, which are attributed to sector $i$.

The sub-species are characterised by the following constraints: first the attribution is required to be complete

$$
\begin{gathered}
\sum_{i=1}^{n} X_{i}=X \\
\sum_{i=1}^{n} Y_{i}=Y \\
\sum_{i=1}^{n} Z_{i}=Z \\
\sum_{i=1}^{n} \tilde{Z}_{i}=\tilde{Z}
\end{gathered}
$$

Second, the sub-species follow the same reaction pathways, 
which is, e.g., for Reactions (R5) and (R6):

$$
\begin{aligned}
& \mathrm{X}_{i}+\mathrm{Y}_{j} \longrightarrow \frac{1}{2} \mathrm{Z}_{i}+\frac{1}{2} \mathrm{Z}_{j}+\mathrm{X}_{i}+\mathrm{Y}_{j} \\
& \mathrm{X}_{i}+\mathrm{Z}_{j} \longrightarrow \mathrm{X}_{i}-\frac{1}{2} \mathrm{Z}_{i}+\frac{1}{2} \mathrm{Z}_{j} \\
& \mathrm{Y}_{i}+\mathrm{Z}_{j} \longrightarrow \mathrm{Y}_{i}-\frac{1}{2} \mathrm{Z}_{i}+\frac{1}{2} \mathrm{Z}_{j}
\end{aligned}
$$

and for $\tilde{Z}$ accordingly. For the Z-production reaction (R11) we consider a molecule $X_{i}$, i.e. a molecule $X$, which has been emitted by source $i$ and a molecule $Y_{j}$, i.e. a molecule $Y$, which has been emitted by source $j$. The product is one molecule $Z$. Since both emission categories $i$ and $j$ are involved equally important, the resulting species are $\frac{1}{2} Z_{i}$ and $\frac{1}{2} Z_{j}$. In the case that a molecule $X_{i}$ reacts with $Y_{i}$, we obtain a molecule $Z_{i}$. For the Z-loss reactions (R12-R13) this consideration is in analogy: When molecules $X$ and $Z$ react, where $X$ and $Z$ are assigned to emission category $i$ and $j$, i.e. $X_{i}$ and $Z_{j}$, then both categories are equally important for the destruction of one $Z$ molecule and the change $-1 Z$ arises from $-\frac{1}{2} Z_{i}-\frac{1}{2} Z_{j}$. Starting from one molecule $Z_{j}$, this results in $-\frac{1}{2} Z_{i}+\frac{1}{2} Z_{j}$ on the left side of Reaction (R12).

From this we can derive the differential equations for the sub-species:

$$
\begin{aligned}
\dot{X}_{i} & =E_{\mathrm{X}, i}-\tau_{\mathrm{X}}^{-1} X_{i} \\
\dot{Y}_{i} & =E_{\mathrm{Y}, i}-\tau_{\mathrm{Y}}^{-1} Y_{i} \\
\dot{Z}_{i} & =P_{\mathrm{Z}, i}\left(X_{i}, Y_{i}\right)-D_{\mathrm{Z}, i}\left(X_{i}, Y_{i}, Z_{i}\right) \\
\dot{\tilde{Z}}_{i} & =P_{\mathrm{Z}, i}\left(X_{i}, Y_{i}\right)-\tilde{D}_{\mathrm{Z}, i}\left(X_{i}, Y_{i}, \tilde{Z}_{i}\right)
\end{aligned}
$$

with $P_{\mathrm{Z}, i}$ and $D_{\mathrm{Z}, i}$ production and loss terms of $\mathrm{Z}_{i}$ with

$$
\begin{aligned}
& P_{\mathrm{Z}, i}\left(X_{i}, Y_{i}\right) \\
& =P_{\mathrm{XY}}\left(X_{i} Y_{i}+\sum_{j \neq i} \frac{1}{2} X_{i} Y_{j}+\sum_{j \neq i} \frac{1}{2} X_{j} Y_{i}\right) \\
& =P_{\mathrm{XY}}\left(X_{i} Y_{i}+\frac{1}{2} X_{i}\left(Y-Y_{i}\right)+\frac{1}{2}\left(X-X_{i}\right) Y_{i}\right) \\
& =\frac{1}{2} P_{\mathrm{XY}}\left(X_{i} Y+X Y_{i}\right) \\
& D_{\mathrm{Z}, i}\left(X_{i}, Y_{i}, Z_{i}\right) \\
& =D_{\mathrm{X}}\left(X_{i} Z_{i}+\frac{1}{2} X_{i}\left(Z-Z_{i}\right)+\frac{1}{2}\left(X-X_{i}\right) Z_{i}\right) \\
& \quad+D_{\mathrm{Y}_{1}}\left(Y_{i} Z_{i}+\frac{1}{2} Y_{i}\left(Z-Z_{i}\right)+\frac{1}{2}\left(Y-Y_{i}\right) Z_{i}\right) \\
& =\frac{1}{2} D_{\mathrm{X}}\left(X_{i} Z+X Z_{i}\right)+\frac{1}{2} D_{\mathrm{Y}_{1}}\left(Y_{i} Z+Y Z_{i}\right)
\end{aligned}
$$

and

$$
\begin{aligned}
& \tilde{D}_{\mathrm{Z}, \mathrm{i}}\left(X_{i}, Y_{i}, \tilde{Z}_{i}\right) \\
& =\frac{1}{2} D_{\mathrm{X}}\left(X_{i} \tilde{Z}+X \tilde{Z}_{i}\right)+D_{\mathrm{Y}_{2}}\left(\frac{1}{3} Y^{2} \tilde{Z}_{i}+\frac{2}{3} Y_{i} Y \tilde{Z}\right)
\end{aligned}
$$

It can easily be shown that

$$
\begin{aligned}
\sum_{i=1}^{n} P_{\mathrm{Z}, i}\left(X_{i}, Y_{i}\right) & =P_{\mathrm{XY}} X Y \\
\sum_{i=1}^{n} D_{\mathrm{Z}, i}\left(X_{i}, Y_{i}, Z_{i}\right) & =D_{\mathrm{X}} X Z+D_{\mathrm{Y}_{1}} Y Z . \\
\sum_{i=1}^{n} \tilde{D}_{\mathrm{Z}, i}\left(X_{i}, Y_{i}, \tilde{Z}_{i}\right) & =D_{\mathrm{X}} X \tilde{Z}+D_{\mathrm{Y}_{2}} Y Y \tilde{Z} .
\end{aligned}
$$

This tagging methodology has two major characteristics: (1) it is invariant and (2) it is convergent. The first point means that for any solutions of Eqs. (1)-(3) and (11)-(13) the constraints (7)-(9) are fullfilled, if this holds for the initial conditions. This can easily be shown with Eqs. (21) and (22).

The second point means that for any two solutions $\left(X_{i}^{1}, Y_{i}^{1}, Z_{i}^{1}\right)$ and $\left(X_{i}^{2}, Y_{i}^{2}, Z_{i}^{2}\right)$ of (11)-(13) with two different initial conditions, the difference in the solutions exponentially converge to zero (see Appendix A).

Figure 1c, d sketches the principle idea behind the tagging, namley that all emissions have the same ozone formation potential indicated by the origin line. Or in other words, molecules, which potentially undergo a certain reaction have all the same probability to undergo this reaction, independent from the emission category. This implies that the break down into categories follows a linear relationship (origin line) for a non-linear chemistry (blue curve).

Note that for simplicity reasons the simple sketch holds only for a well mixed zero-dimensional box model chemistry. Emitted species experience very different chemical conditions, which cannot be visualized in a simple sketch.

For applications in real chemistry schemes, the tagging method is in principle not different from the described one. To each species, $n$ (number of regarded emission categories) tagged species are associated. For each of this tagged species, production and loss terms have to be deduced. This decomposition of the production and loss terms into the contributions from individual emission categories is essential to the tagging methodology. This is a combinatorical problem, which can be solved in analogy to the above mentioned cases for 2 and 3-body reactions. A general approach is given in Appendix B. However, since the tagging of a whole chemical system is likely to be too computational demanding a mapping of the complex chemical system, including the production and loss terms, onto a simpler family concept might be helpful. Then only the families need to be tagged (Grewe, 2004).

\subsection{Contributions by pairs of simulations (sensitivity method)}

Most studies, which concentrate on the impact of a certain emission on the composition, derive the contribution of the emission category to the concentration of a species (e.g. ozone) by comparing two simulations, one simulation 
with all emissions and one simulation with a perturbation of the respective emission category (sensitivity method). An overview is given in a sketch (Fig. 1), which shows the ozone response to a certain $\mathrm{NO}_{\mathrm{x}}$ emission. (Since there is basically a monotonic relationship between the $\mathrm{NO}_{\mathrm{x}}$ emission and concentration, this can also be regarded as concentrations.) Two simulation results are shown with stars, representing a base case (blue) and a perturbation simulation (red).

Mathematically, this approach is based on a Taylor approximation of the regarded quantity $f$ as a function of the emissions around a base case, i.e. the case where all emissions $\left(e_{0}\right)$ are employed:

$$
\begin{aligned}
f\left(e_{0}+\alpha e^{\mathrm{c}}\right) & \approx f\left(e_{0}\right)+\alpha e^{\mathrm{c}} f^{\prime}\left(e_{0}\right) \\
& =\tilde{f}\left(e_{0}+\alpha e^{\mathrm{c}}\right),
\end{aligned}
$$

where $e^{\mathrm{c}}$ denotes a certain emission category and $\alpha \in[-1,1]$ the strength of the perturbation. The case $\alpha=-1$ represents the situation, where all emissions from the respective category are excluded. $f^{\prime}\left(e_{0}\right)$ (black dashed line in Fig. 1a), the derivative, is the efficiency of the production of the regarded species per emission. Considering the contribution of a specific emission to the concentration of $f$ with this approach implies that all categories experience the same production efficiency, since the derivative $f^{\prime}$ is evaluated at $e_{0}$.

The contribution $\delta f$ of a certain emission category $e^{\mathrm{c}}$ is then

$$
\begin{aligned}
\delta f & =\tilde{f}\left(e_{0}+e^{\mathrm{c}}\right)-\tilde{f}\left(e_{0}\right) \\
& =e^{\mathrm{c}} f^{\prime}\left(e_{0}\right) .
\end{aligned}
$$

The main focus is now to determine the derivative $f^{\prime}$. This can be done by a pair of simulations, one with all emissions and one with a perturbation of an emission category, with $\Delta^{\alpha} f$ (green line in Fig. 1a) the difference in $f$ between both simulations:

$$
\begin{aligned}
f^{\prime}\left(e_{0}\right) & \approx \frac{f\left(e_{0}\right)-f\left(e_{0}+\alpha e^{\mathrm{c}}\right)}{e_{0}-\left(e_{0}+\alpha e^{\mathrm{c}}\right)} \\
& =\left(f\left(e_{0}\right)-f\left(e_{0}+\alpha e^{\mathrm{c}}\right)\right) \frac{-1}{\alpha e^{\mathrm{c}}} \\
& =-\frac{\Delta^{\alpha} f}{\alpha e^{\mathrm{c}}}
\end{aligned}
$$

where $\Delta^{\alpha} f$ is the difference in two simulations. The smaller $\alpha$ the less different is the chemical background in the two simulations, but the more difficult it will be to obtain a statistical robust perturbation of $f$. Within QUANTIFY a value $\alpha=-0.05$ has been selected (Hoor et al., 2009). Other modelling studies used also other values, e.g. $+30 \%$ (Isaksen, 2003), $+5 \%$ (Grewe, 2004), $-20 \%$ and $-100 \%$ (Wu et al., 2009; Fiore et al., 2009).

Furthermore, a small $\alpha$, which guarantees that the chemical background is comparable in the simulations, also guarantees that the estimated contributions from different sectoral emissions are consistently calculated and thereby comparable. In Sect. 6 we will introduce an indicator, which tests the consistency and comparability in the contribution calculations based on the sensitivity methods for global chemistry models. We call this indicator: error $\epsilon_{\alpha}$ (see also Fig. 1f).

We obtain for the estimated contribution $\delta^{\alpha} f$ :

$\delta^{\alpha} f=-\frac{\Delta^{\alpha} f}{\alpha}$

Note that the calculation of the contribution is mathematically a scaling of the difference of two model simulations in which an emission source is scaled by the value $-\alpha^{-1}$. However, conceptually, $\alpha$ is only used to calculate most accurately the derivative, which is then multiplied by the total emission of the respective source (Eq. 27).

In Fig. 1f two emission categories for $\mathrm{NO}_{\mathrm{x}}$ are considered $\left(\mathrm{NO}_{\mathrm{x}}-1\right.$ : light blue and $\mathrm{NO}_{\mathrm{x}}-2$ : red) and the results for $\delta^{\alpha} \mathrm{O}_{3}$ are indicated as vertical lines with the respective colour. Simply from the sketch it is already obvious that the sum of the contributions $\left(\mathrm{O}_{3}-1+\mathrm{O}_{3}-2\right)$ does not equal the actual ozone contribution and an error (orange) remains, which is due to the non linear response of $\mathrm{O}_{3}$ to $\mathrm{NO}_{\mathrm{x}}$ emissions. As a consequence of this non-linearity, the tangent to the ozone curve in $e_{0}$ is affine linear, i.e. it has, in general, a $y$-intercept. This $y$-intercept is the part of the ozone concentration, which cannot be explained by the sensitivity method. Therefore this method exhibits a principle error.

In global chemistry simulations this error applies only to the ozone fraction, which is produced by tropospheric chemistry. In Sect. 6 we demonstrate how to estimate this error, which we call $\epsilon_{\beta}$ in global chemistry simulations (see also Fig. 1f).

To summarize, the sensitivity method is in principle inappropriate for source attribution, but well suited to address impacts of e.g. future emission policies.

\section{Steady-state solutions}

In order to investigate the impact of a specific emission on the concentration of a species, chemistry-climate or chemistry transport models are run in a quasi-equilibrium state. Here, we are considering the same approach and concentrate on the steady-state solutions ( $X^{\mathrm{eq}}, Y^{\mathrm{eq}}, Z^{\mathrm{eq}}, \tilde{Z}^{\mathrm{eq}}$ ) of Eqs. (1) to (4) and the respective solutions $X_{i}^{\mathrm{eq}}, Y_{i}^{\mathrm{eq}}, Z_{i}^{\mathrm{eq}}, \tilde{Z}_{i}^{\mathrm{eq}}$, for (11) to (14), hence the left side equals zero:

$$
\begin{aligned}
X^{\mathrm{eq}} & =E_{\mathrm{X}} \tau_{\mathrm{X}} \\
Y^{\mathrm{eq}} & =E_{\mathrm{Y}} \tau_{\mathrm{Y}} \\
Z^{\mathrm{eq}} & =\frac{P_{\mathrm{XY}} X^{\mathrm{eq}} Y^{\mathrm{eq}}}{D_{\mathrm{X}} X^{\mathrm{eq}}+D_{\mathrm{Y}_{1}} Y^{\mathrm{eq}}} \\
& =\frac{P_{\mathrm{XY}} E_{\mathrm{X}} \tau_{\mathrm{X}} E_{\mathrm{Y}} \tau_{\mathrm{Y}}}{D_{\mathrm{X}} E_{\mathrm{X}} \tau_{\mathrm{X}}+D_{\mathrm{Y}_{1}} E_{\mathrm{Y}} \tau_{\mathrm{Y}}} \\
\tilde{Z}^{\mathrm{eq}} & =\frac{P_{X Y} X^{\mathrm{eq}} Y^{\mathrm{eq}}}{D_{\mathrm{X}} X^{\mathrm{eq}}+D_{\mathrm{Y}_{2}} Y^{\mathrm{eq}} Y^{\mathrm{eq}}}
\end{aligned}
$$


Table 2. Information on chosen reaction rates for the two chemical reaction systems.

\begin{tabular}{rllcl}
\hline \multicolumn{1}{r}{ Reaction } & & \multicolumn{2}{c}{ Rate Constant } \\
\cline { 3 - 5 } & & & {$\left[\mathrm{cm}^{3} \mathrm{molec}^{-1} \mathrm{~s}^{-1}\right]$} & {$\left[\mathrm{ppbv}^{-1} \mathrm{~s}^{-1}\right]$} \\
\hline $\mathrm{X}+\mathrm{Y}$ & $\longrightarrow$ & $\mathrm{Z}$ & $3.5 \times 10^{-14}$ & $=8.9 \times 10^{-4}$ \\
$\mathrm{X}+\mathrm{Z}$ & $\longrightarrow$ & $\mathrm{X}$ & $1.0 \times 10^{-14}$ & $=2.5 \times 10^{-4}$ \\
$\mathrm{Y}+\mathrm{Z}$ & $\longrightarrow$ & $\mathrm{Y}$ & $1.0 \times 10^{-14}$ & $=2.5 \times 10^{-4}$ \\
\hline $\mathrm{X}+\mathrm{Y}$ & $\longrightarrow$ & $\tilde{\mathrm{Z}}$ & $3.5 \times 10^{-14}$ & $=8.9 \times 10^{-4}$ \\
$\mathrm{X}+\tilde{\mathrm{Z}}$ & $\longrightarrow$ & $\mathrm{X}$ & $1.0 \times 10^{-14}$ & $=2.5 \times 10^{-4}$ \\
$\mathrm{Y}+\mathrm{Y}+\tilde{\mathrm{Z}}$ & $\longrightarrow$ & $\mathrm{Y}+\mathrm{Y}$ & $3.4 \times 10^{-27} *$ & $=2.5 \times 10^{-2} *$ \\
\hline
\end{tabular}

* the units differ for this reaction, since it is a three-body reaction: $\mathrm{cm}^{6} \mathrm{molec}^{-2} \mathrm{~s}^{-1}$ and $\mathrm{ppbv}^{-2} \mathrm{~s}^{-1}$, respectively.

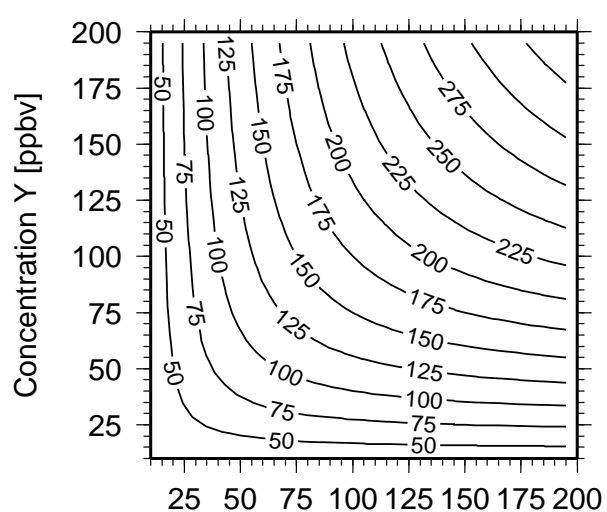

Concentration $X[p p b v]$

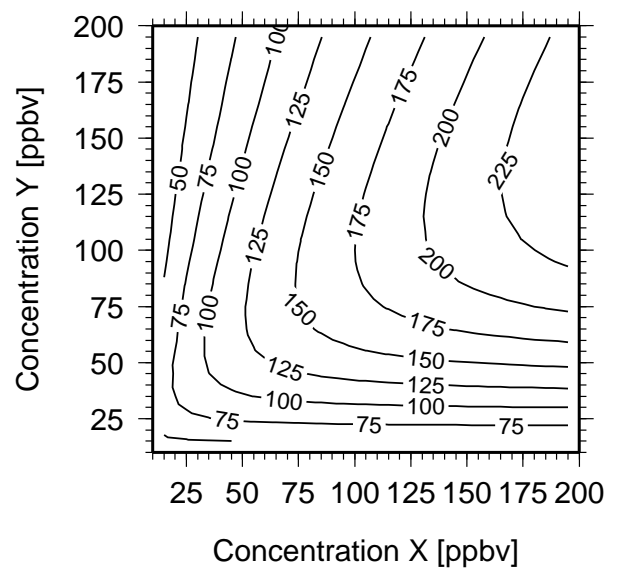

Fig. 2. Equilibrium concentrations for species $Z$ (top) and $\tilde{Z}$ (bottom) [ppbv] as a function of the concentrations of $\mathrm{X}$ and $\mathrm{Y}$.

We consider reaction rates for (R5)-(R7) (Table 2). The equilibrium concentrations ( $Z^{\text {eq }}$ and $\left.\tilde{Z}^{\text {eq }}\right)$ are shown in Fig. 2. They show typical ozone characteristics: for a certain relation between the precursors $\mathrm{X}$ and $\mathrm{Y}$, the equilibrium concentration of $\mathrm{Z}$ is maximum. The concentration increases only slightly, when only one of either concentration

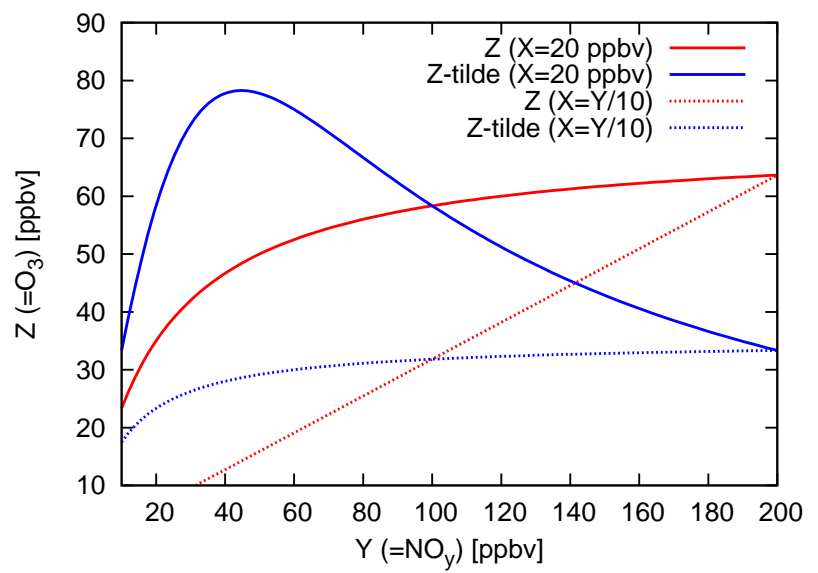

Fig. 3. Equilibrium concentrations for $Z$ (red line) and $\tilde{Z}$ (blue line) [ppbv] as a function of the concentration of $\mathrm{Y}$ for a constant concentration of $\mathrm{X}=20 \mathrm{ppbv}$ (solid) and a constant ratio between the concentration of species $\mathrm{X}$ and $\mathrm{Y}$ of 1:10.

$X$ and $Y$ is further increased. This represents a $\mathrm{X}(\mathrm{VOC})$ and $\mathrm{Y}\left(\mathrm{NO}_{\mathrm{x}}\right)$ limited region (Seinfeld and Pandis, 2006). The equilibrium concentrations of $\tilde{Z}$ shows an additional feature: for increasing concentrations of $\mathrm{Y}\left(\mathrm{NO}_{\mathrm{x}}\right)$ the destruction of $\tilde{Z}$ is increasing more strongly, leading to a decrease, which can be observed for ozone in $\mathrm{NO}_{\mathrm{x}}$ rich environments (ozone titration). Figure 3 shows the concentration of $Z$ (red line) and $\tilde{Z}$ (blue line) as a function of the concentration $Y$ for two cases: a constant $X$ concentration $(X=20 \mathrm{ppbv}$, solid lines) and constant ratio between the $X$ and $Y$ concentration ( $X=Y / 10$, dotted lines). Clearly, for a constant concentration of $X$, the concentration of $Z$ steadily increases, however with a very small rate for high $Y$ concentrations (X limited region), whereas $\tilde{Z}$ shows a decrease for $\mathrm{Y}$ concentration larger than approximately $40 \mathrm{ppbv}$ (titration effect). Both chemical systems also show a very different behaviour, when the ratio between the precursors $\mathrm{X}$ and $\mathrm{Y}$ is constant (dotted line). In this case, the concentration of $\mathrm{Z}$ increases linearly, whereas the concentration of $\tilde{Z}$ shows a saturation effect (X limited region). Since the equation for $Z$ (3) describes a cone, a constant ratio refers to an edge of the cone. And hence the systems have two different degrees of linearity. Mathematically, this can be described by:

$\nabla Z^{\mathrm{eq}}\left(\begin{array}{l}X^{\mathrm{eq}} \\ Y^{\mathrm{eq}}\end{array}\right)=Z^{\mathrm{eq}}$

$\nabla \tilde{Z}^{\mathrm{eq}}\left(\begin{array}{c}X^{\mathrm{eq}} \\ Y^{\mathrm{eq}}\end{array}\right)=\tilde{Z}^{\mathrm{eq}} \frac{D_{\mathrm{X}} X^{\mathrm{eq}}}{D_{\mathrm{X}} X^{\mathrm{eq}}+D_{\mathrm{Y}_{2}} Y^{\mathrm{eq}} Y^{\mathrm{eq}}}<\tilde{Z}^{\mathrm{eq}}$

Therefore the second chemical system (Eq. 38) does not show any linearity in contrast to the first chemical system (Eq. 37). The smallest deviations from linearity occur for large $X^{\text {eq }}$ and small $Y^{\mathrm{eq}}$.

Figure 4 describes for a constant background situation $(X=20 \mathrm{ppbv}$ and $Z=40 \mathrm{ppbv})$ the net $Z$ and $\tilde{Z}$ production rates. In the first chemical system the net-production 
term increases linearly with increasing concentration of $\mathrm{Y}$, whereas the net production of $\tilde{Z}$ decreases for large concentrations of $Y$ leading to an effective depletion of $\tilde{Z}$. The shape of the net-production is similar to the ozone response to increasing concentrations of $\mathrm{NO}_{\mathrm{x}}$ (Ehhalt and Rohrer, 1994).

To summarise, both atmospheric reaction systems represent the main characteristics of observed tropospheric ozone chemistry. They cannot replace a detailed chemical calculation nor can they be used to interpret observational data. But they are simple enough to be solved analytically and hence can be used as test cases for diagnostic methods.

For the tagged species steady-state solutions can easily be derived:

$$
\begin{aligned}
X_{i}^{\mathrm{eq}} & =E_{\mathrm{X}, i} \tau_{\mathrm{X}} \\
Y_{i}^{\mathrm{eq}} & =E_{\mathrm{Y}, i} \tau_{\mathrm{Y}} \\
Z_{i}^{\mathrm{eq}} & =\frac{a_{i}}{c}-\frac{b_{i}}{c} Z^{\mathrm{eq}} \text { with } \\
a_{i} & =P_{\mathrm{XY}} \tau_{\mathrm{X}} \tau_{\mathrm{Y}}\left(E_{\mathrm{X}, i} E_{\mathrm{Y}}+E_{\mathrm{X}} E_{\mathrm{Y}, i}\right) \\
& =P_{\mathrm{XY}}\left(X_{i}^{\mathrm{eq}} Y^{\mathrm{eq}}+X^{\mathrm{eq}} Y_{i}^{\mathrm{eq}}\right) \\
b_{i} & =D_{\mathrm{X}} E_{\mathrm{X}, i} \tau_{\mathrm{X}}+D_{\mathrm{Y}_{1}} E_{\mathrm{Y}, i} \tau_{\mathrm{Y}} \\
& =D_{\mathrm{X}} X_{i}^{\mathrm{eq}}+D_{\mathrm{Y}_{1}} \mathrm{Y}_{i}^{\mathrm{eq}} \\
c & =D_{\mathrm{X}} E_{\mathrm{X}} \tau_{\mathrm{X}}+D_{\mathrm{Y}_{1}} E_{\mathrm{Y}} \tau_{\mathrm{Y}} \\
& =D_{\mathrm{X}} X^{\mathrm{eq}}+D_{\mathrm{Y}_{1}} Y^{\mathrm{eq}} \\
\tilde{Z}_{i}^{\mathrm{eq}} & =\frac{a_{i}}{\tilde{c}}-\frac{\tilde{b}_{i}}{\tilde{c}} \tilde{Z}^{\mathrm{eq}} \text { with }^{\mathrm{eq}} \\
\tilde{b}_{i} & =D_{\mathrm{X}} X_{i}^{\mathrm{eq}}+\frac{4}{3} D_{\mathrm{Y}_{2}} \mathrm{Y}_{i}^{\mathrm{eq}} Y^{\mathrm{eq}} \\
\tilde{c} & =D_{\mathrm{X}} X^{\mathrm{eq}}+\frac{2}{3} D_{\mathrm{Y}_{2}} Y^{\mathrm{eq}} Y^{\mathrm{eq}}
\end{aligned}
$$

Steady-state solutions for $\delta_{i}^{\alpha} X, \delta_{i}^{\alpha} Y, \delta_{i}^{\alpha} Z$ (Eqs. 32-34) can easily be derived by inserting the solutions for $X^{\mathrm{eq}}$ and $Y^{\mathrm{eq}}$ for the 2 regarded emission scenarios in Eq. (31):

$$
\begin{aligned}
\delta_{i}^{\alpha} X= & E_{\mathrm{X}, i} \tau_{\mathrm{X}}=X_{i}^{\mathrm{eq}} \\
\delta_{i}^{\alpha} Y= & E_{\mathrm{Y}, i} \tau_{\mathrm{Y}}=Y_{i}^{\mathrm{eq}} \\
\delta_{i}^{\alpha} Z= & \frac{a_{i}}{c+\alpha b_{i}}+\frac{\alpha P_{\mathrm{XY}} \tau_{\mathrm{X}} \tau_{\mathrm{Y}} E_{\mathrm{X}, i} E_{\mathrm{Y}, i}}{c+\alpha b_{i}} \\
& -\frac{b_{i} P_{\mathrm{XY}} \tau_{\mathrm{X}} \tau_{\mathrm{Y}} E_{\mathrm{X}} E_{\mathrm{Y}}}{c(c+\alpha b)} \\
= & \frac{a_{i}+\alpha d_{i}}{c+\alpha b_{i}}-\frac{b_{i}}{c+\alpha b_{i}} Z^{\mathrm{eq}}, \text { with } \\
d_{i}= & P_{\mathrm{XY}} \tau_{\mathrm{X}} \tau_{\mathrm{Y}} E_{\mathrm{X}, i} E_{\mathrm{Y}, i} \text { and } \\
\delta_{i}^{\alpha} \tilde{Z}= & \frac{a_{i}+\alpha d_{i}}{\tilde{c}+\tilde{h}}-\frac{\tilde{b}_{i}-\tilde{g}_{i}}{\tilde{c}+\tilde{h}+\alpha\left(\tilde{b}_{i}-\tilde{g}_{i}\right)} \tilde{Z}^{\mathrm{eq}}, \text { with } \\
\tilde{g}_{i}= & \frac{2}{3} D_{\mathrm{Y}_{2}} Y_{i}^{\mathrm{eq}} Y^{\mathrm{eq}}-\alpha D_{\mathrm{Y}_{2}} Y_{i}^{\mathrm{eq}} Y_{i}^{\mathrm{eq}} \\
\tilde{h}= & \frac{1}{3} D_{\mathrm{Y}_{2}} Y^{\mathrm{eq}} Y^{\mathrm{eq}} .
\end{aligned}
$$

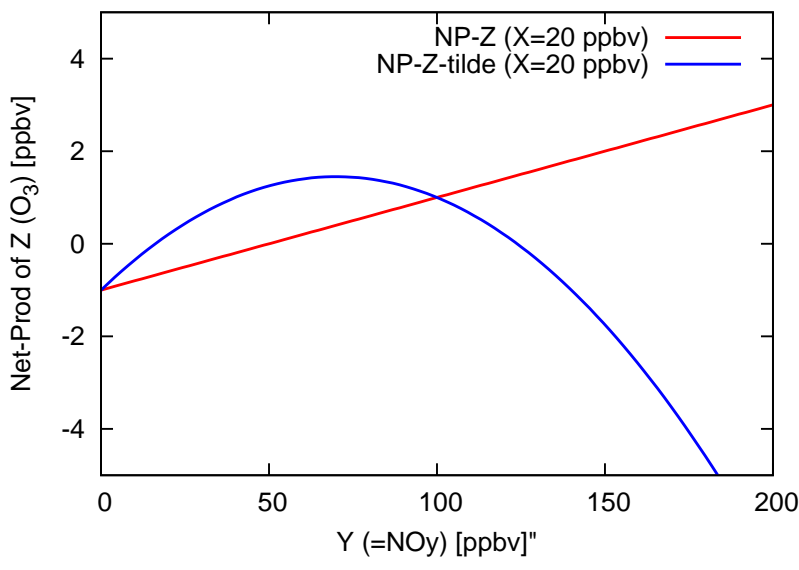

Fig. 4. Net production rates [ppbv/s] for a constant concentration of $\mathrm{Z}$ (red line) and $\tilde{\mathrm{Z}}$ (blue line) of $40 \mathrm{ppbv}$ and a mixing ratio of $X=20 \mathrm{ppbv}$.

Therefore the results from both methods (Sects. 3.2 and 3.3) agree for the species $\mathrm{X}$ and $\mathrm{Y}$, whereas they normally differ for species $\mathrm{Z}$ and $\tilde{Z}$. However, the solutions converge for small $\alpha$ :

$\delta_{i}^{\alpha} Z \stackrel{\alpha \rightarrow 0}{\longrightarrow} Z_{i}^{\mathrm{eq}}$

whereas they do not converge in general for the second chemical system:

$$
\begin{aligned}
\delta_{i}^{\alpha} \tilde{Z} & \stackrel{\alpha \rightarrow 0}{\longrightarrow} \frac{a_{i}}{\tilde{c}+\tilde{h}}-\frac{\tilde{b}_{i}-\frac{2}{3} D_{\mathrm{Y}_{2}} Y_{i}^{\mathrm{eq}} Y^{\mathrm{eq}}}{\tilde{c}+\tilde{h}} \tilde{Z}^{\mathrm{eq}} \\
& \neq \tilde{Z}_{i}^{\mathrm{eq}} .
\end{aligned}
$$

This also implies that

$$
\begin{aligned}
& \sum_{i=1}^{n} X_{i}^{\mathrm{eq}}=\sum_{i=1}^{n} \delta_{i}^{\alpha} X=X^{\mathrm{eq}} \\
& \sum_{i=1}^{n} Y_{i}^{\mathrm{eq}}=\sum_{i=1}^{n} \delta_{i}^{\alpha} Y=Y^{\mathrm{eq}} \\
& \sum_{i=1}^{n} Z_{i}^{\mathrm{eq}}=Z^{\mathrm{eq}}, \text { but } \\
& \sum_{i=1}^{n} \delta_{i}^{\alpha} Z \neq Z^{\mathrm{eq}} \text { in general, and } \\
& \sum_{i=1}^{n} \delta_{i}^{\alpha} \tilde{Z} \neq \tilde{Z}^{\mathrm{eq}} \text { in general. }
\end{aligned}
$$

The last two equations clearly show that the method of determining contributions of emissions to trace gases by pairs of simulations is not able to consistently decompose a concentration into contributions from individual sources, even for the simpler chemistry considered here. 


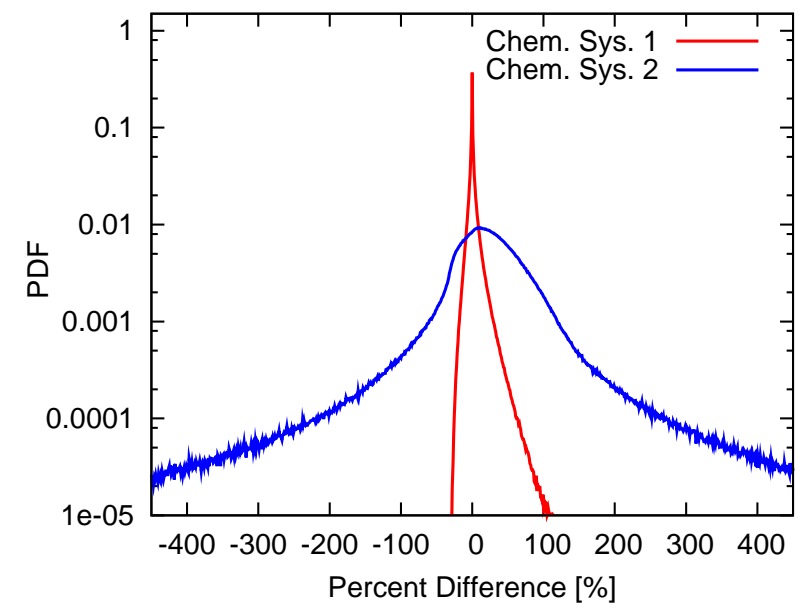

Fig. 5. Probability density function for the errors of the contribution calculation based in the sensitivity method: error $E$ (red) and $\tilde{E}$ (blue) [\%], i.e. for the first and second chemical system. A range of values for $X, Y, X_{i}, Y_{i}$, and $\alpha$ is taken into account with a MonteCarlo Simulation. See text for details.

\section{Error analysis}

In the previous sections, we have revealed that the methodology of calculating the contributions from individual sources to concentration changes by pairs of simulations leads to a potentially large error. In this section we actually calculate this error for the two chemical systems, which we presented in Sect. 2. Figure 5 shows the probability density function (PDF) for the relative errors $E$ (red) and $\tilde{E}$ (blue), i.e.

$E=\frac{\delta_{i}^{\alpha} Z-Z_{i}}{Z_{i}} \cdot 100 \%$

$\tilde{E}=\frac{\delta_{i}^{\alpha} \tilde{Z}-\tilde{Z}_{i}}{\tilde{Z}_{i}} \cdot 100 \%$

The PDF is derived with a Monte Carlo simulation covering the parameter ranges between 10 and $200 \mathrm{ppbv}$ for $X$ and $Y$, fractions for $X_{i}$ and $Y_{i}$ between 5\% and 95\% and values of $\alpha$ ranging between -100 and $100 \%$. Clearly, the error for the first chemical system is close to zero for most cases (note the logarithmic scale) and less than a factor of two for all except a very few cases. In contrast, the second chemical system, which is characterised by a stronger non-linearity, reveals a much broader PDF, i.e. there is a large probability for large errors, e.g. the probability that the error $|\tilde{E}|$ is larger than $50 \%$ is $35.6 \%$ for the second chemical system compared to $0.4 \%$ for the first system.

The errors are dependent on the choice of $\alpha$, as discussed in Sect. 4 and Eq. (60) and converge to zero for decreasing $\alpha$, at least for the first chemical system. This convergence is shown in Fig. 6a, where the error probability decreases almost to zero for values of $|\alpha|$ decreasing from 100 to $5 \%$. Generally the relative error is larger for negative perturbations, i.e. negative $\alpha$, than for positive perturbations.
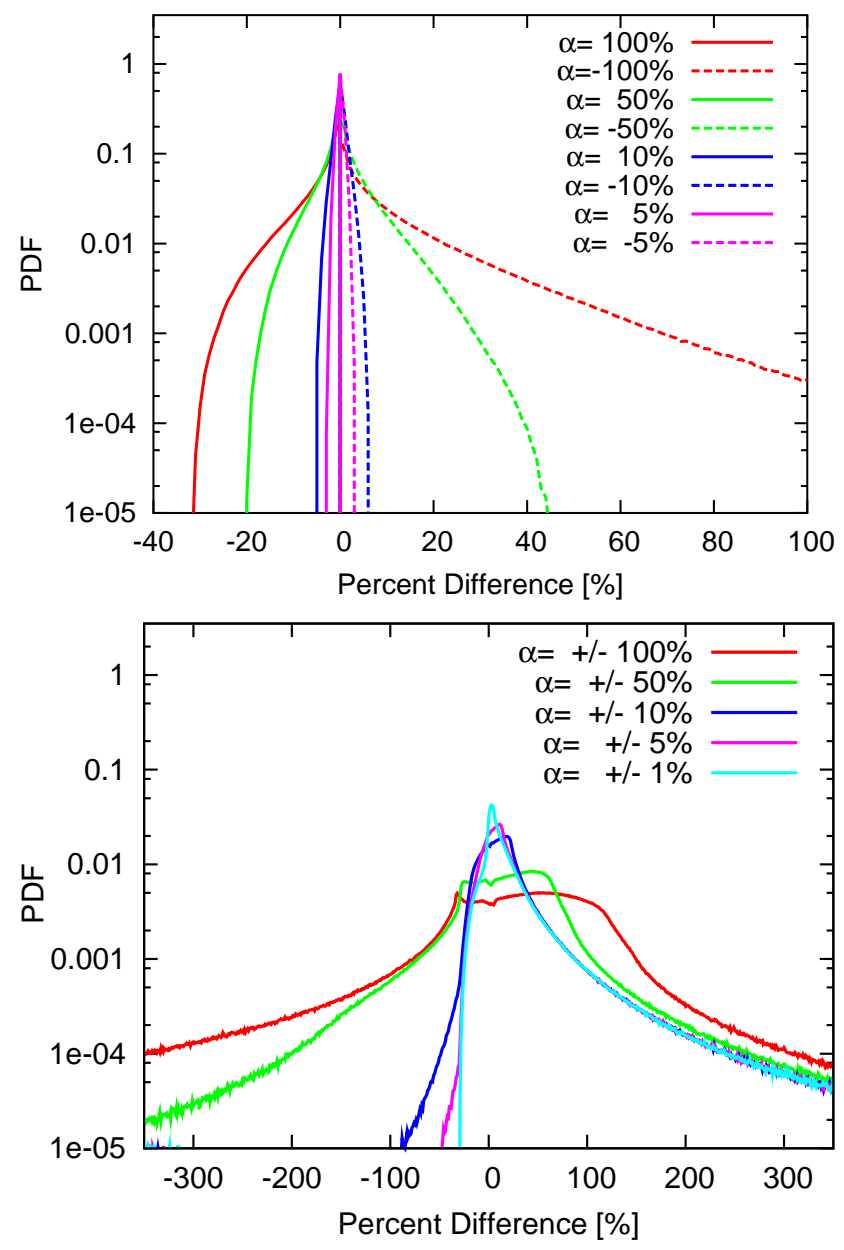

Fig. 6. Probability density function for the error [\%] for specific values of $\alpha$ for the first chemical system (top) and the second chemical system (bottom).

In contrast, only a small reduction of the errors can be found for the second chemical system (Fig. 6b). However, this result largely depends on two conditions: first, the degree of non-linearity of the chemical system and second on the degree of the deviation of expression (38) from equality. For small concentrations of $\mathrm{X}$ and $\mathrm{Y}$ both chemical systems show a quite linear behaviour (Fig. 3). If additionally the concentration $Y$ is much smaller than the concentration of $\mathrm{X}$ then both conditions have a much smaller impact (Fig. 7) and the errors $E$ and $\tilde{E}$ show a more similar behaviour.

Although the shapes of the functions $Z$ and $\tilde{Z}$ are comparable for small concentrations of $\mathrm{X}$ and $\mathrm{Y}$ and the probability of a small error is large, the mean value of the errors (not shown) and the standard deviation (Fig. 8) are large for the second chemical system (bottom). The mean value of the error represents a bias, which might be corrected. However, if the standard deviation is large then the method of calculating the contributions from emission categories to the concentration of $\mathrm{Z}$ largely depends on the fraction $X_{i}$ and $Y_{i}$ as well as 


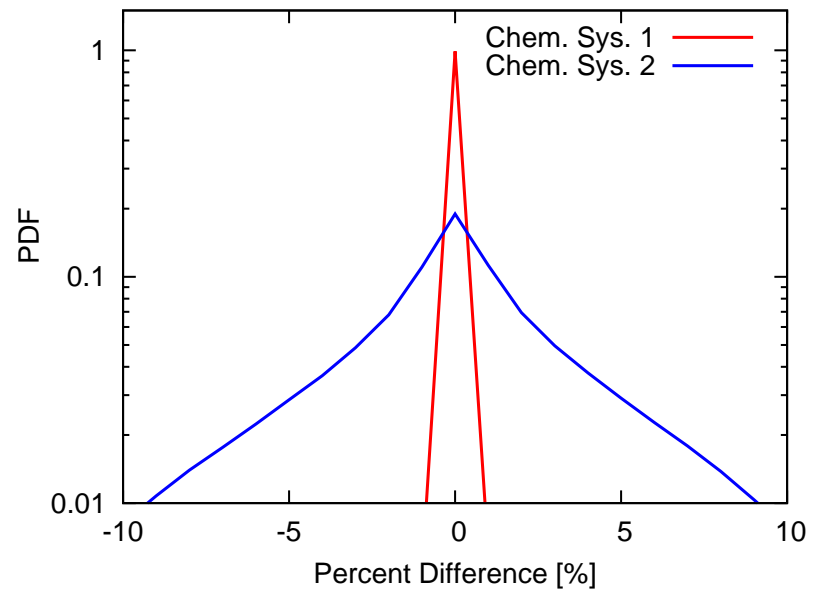

Fig. 7. Probability density function for the errors $E$ (red) and $\tilde{E}$ (blue) [\%] for specific settings of the concentrations $X$ and $Y$, which minimise the errors: $10 \mathrm{ppbv} \leq X \leq 20 \mathrm{ppbv}$; $0.1 \mathrm{ppbv} \leq Y \leq 1 \mathrm{ppbv}$.

the choice of $\alpha$. The gradient of $Z$ is estimated by a difference method, which depends on $\alpha$ (see discussion Sect. 3.3), but also on $\left(X_{i}, Y_{i}\right)$, which is used to calculate the perturbation. Therefore, although the two systems show similarities in certain regions, which leads to an agreement in the most probable error (Fig. 7), the total error characteristic is very different. It turns out that both, the mean error (not shown) and the standard deviation (Fig. 8) are minimal in regions where both concentrations $X$ and $Y$ are comparable, and no large curvature occurs.

\section{Implications and recommendations for attribution studies}

Our analysis is based on (a) simplified chemical systems and (b) a zero dimensional box model. Here, we give some indications how our results can be used for global chemistry simulations. The contributions from a source to, e.g., the ozone concentration by pairs of simulations (see Sect. 3) have uncertainties, which we address here. For simplicity reasons, we concentrate on ozone. In general, this can be applied to any species. And further, we take as examples the studies by Hoor et al. (2009) and Grewe (2004).

In Sect. 3.3, we discussed two types of errors (which we denote $\epsilon_{\alpha}$ and $\epsilon_{\beta}$ ) in the determination of the attribution of species to emission categories $\left(=\delta_{i}^{\alpha}\right)$ with the methodology employing pairs of simulations. First the accuracy of the determination of the contribution $\delta_{i}^{\alpha}$ depends on $\alpha$, via the estimation of the gradient of the respective species (here $\mathrm{Z}$ ). Note, that we found a convergence of the attribution methodology by pairs of simulations to the real solution defined by the tagging methodology for the first and more linear chemical system. We now focus on global chemistry simulations. Obviously, the smaller $\alpha$ the more accurate is the calcula-
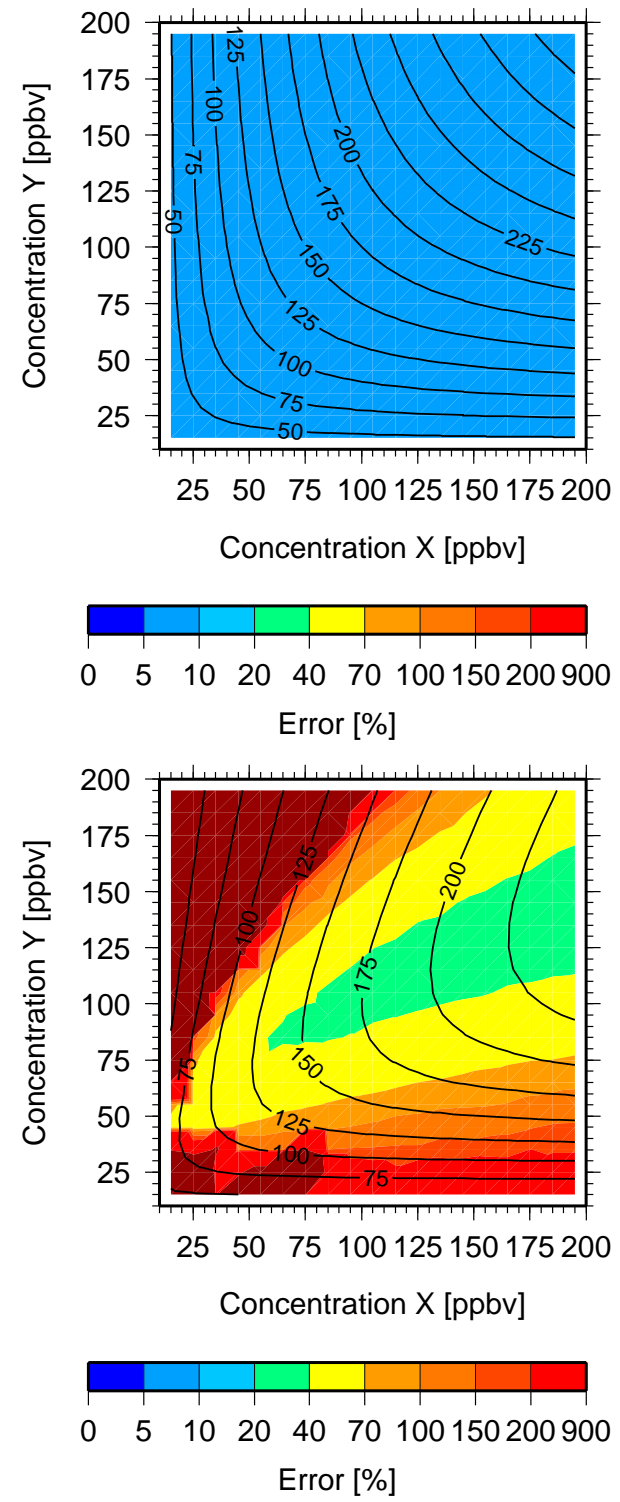

Fig. 8. Standard deviation of the error $E$ (top) and $\tilde{E}$ (bottom) [\%]. Overlaid are the contour lines of the values of $Z$ and $\tilde{Z}$, respectively.

tion of the gradient of ozone. On the other hand the statistical significance of the results decreases greatly if $\alpha$ is too small. And hence the best choice of $\alpha$ is a tradeoff between accuracy in the determination of the gradient and detection of a significant result. In practice, a good indicator whether $\alpha$ has been chosen appropriately is to test, if the sum of individual contributions $\delta_{i}^{\alpha}(i=1, \ldots, k)$ equals the contribution of the sum of the emissions $\left(e_{K}=e_{1}+\ldots+e_{k}\right)$ from categories 1 to $k\left(=\delta_{K}^{\alpha}\right)$, which is a necessary, but not sufficient condition. An error estimate $\epsilon_{\alpha}$ can be given by:

$\epsilon_{\alpha}=\frac{\sum_{i=1}^{k} \delta_{i}^{\alpha} Z-\delta_{K}^{\alpha} Z}{\delta_{K}^{\alpha} Z}$. 
This type of error was investigated in Hoor et al. (2009) by the intercomparison of the results from their experiments "ROAD", "SHIP", and "AIR" with results of their experiment "ALL". Based on their Table 5, we calculate global errors $\epsilon_{\alpha}$ for two atmospheric regions: the lower atmosphere $(1000-800 \mathrm{hPa})$ and the upper troposphere $(300-200 \mathrm{hPa})$ of $0 \%$ and $0.6 \%$, respectively. Therefore, their choice of $\alpha=5 \%$ was well chosen to derive a good estimate of the gradient of ozone by pairs of simulations.

In Fig. If this error indicates whether the estimated derivative (green line) is equal for all calculated cases, which implies that the change in the background ozone chemistry between the base case and the perturbation simulation is irrelevant.

The second error $\epsilon_{\beta}$ is based on the completeness of the decomposition of the species $\mathrm{Z}$ into the contributions by the individual categories, i.e. a measure on how large the expressions (66) and (67) deviate from equality:

$\epsilon_{\beta}=\frac{\delta_{N}^{\alpha} Z-\left(Z-Z^{\text {strat }}\right)}{\left(Z-Z^{\text {strat }}\right)}$,

where $\delta_{N}^{\alpha} Z$ is, similar to $\delta_{K}^{\alpha} Z$, the contribution of all emissions to the concentration of $Z$ derived with the method of "pairs of simulations" for all $(=N)$ emissions and $Z^{\text {strat }}$ is the contribution of other sources than those considered. For ozone this is the contribution from the stratosphere, which is not included in the simplified chemical systems. However, this has to be included when discussing the consequences for global tropospheric chemistry simulations. In Fig. 1f the error $\epsilon_{\beta}$ is indicated by the orange vertical line. The error $\epsilon_{\beta}$ is independent from the contribution of stratospheric ozone. The error $\epsilon_{\beta}$ is associated with the contribution calculation of the part of the ozone concentration, which is produced by tropospheric chemistry, i.e. by emissions of $\mathrm{NO}_{\mathrm{x}}$, etc. This tropospheric ozone is equal to $Z-Z^{\text {strat }}$. In general, it does not equal the sum of all contributions for the individual sources, because the tangent has a significant y-intercept (Fig. 1f). In the case of $\epsilon_{\alpha}$ equals 0 , the error $\epsilon_{\beta}$ describes the theoretical error of the sensitivity method based on the exact tangent (dashed line). The error $\epsilon_{\beta}$ is independent from the error $\epsilon_{\alpha}$, which represents the fraction of the ozone concentration between the two y-intercepts of the tangent (black line) and the estimated tangent (green line).

Grewe (2004) investigated this error (Eq. 6 and following text in that paper) and found maximum errors in the troposphere of $40 \%$ and $-5 \%$ in the tropopause region. That implies a rescaling of the results derived for $\delta_{i}^{\alpha}$ by $\frac{1}{1-\epsilon_{\beta}}$ to obtain a complete decomposition of the species $\mathrm{Z}$ into their contributions. Or in other words the methodology to calculate the contributions with pairs of simulations underestimates the contributions of tropospheric emissions on ozone in the order of 5 to $25 \%$, assuming that the respective mean $\epsilon_{\beta}$ is in the order of 5 to $20 \%$.
It has to be clearly mentioned that the errors $\epsilon_{\alpha}$ and $\epsilon_{\beta}$ are only estimates based on global averages. Both locally and temporally (e.g. for different seasons) this can vary: $\mathrm{Wu}$ et al. (2009) investigated the impact of $20 \%$ versus $100 \%$ local emission reductions for various source and receptor regions and found no differences in summer but large decrepancies in other seasons and also large differences for different source regions. Additionally, individual contributions can have larger errors, which may compensate when adding. Although the errors may vary with time and location, the method underestimates the contributions, as long as the atmospheric chemistry acts like the second chemical system, i.e. Fig. 2 (bottom). This can be deduced from Eq. (38), where the left side represents the contribution from all sources and the right side the actual concentration.

To summarize, it has to be noted that the sensitivity method is in principle inappropriate for source attribution, but well suited to address impacts of e.g. future emission policies. Therefore, we recommend to use a tagging methodology for deriving contributions from emissions to species. However, a full implementation of a tagging method as introduced in this paper was, to our knowledge, not implemented in any global chemistry model. Further, a full tagging method is to computational demanding, since it amplifies the whole chemistry scheme, if not mapped onto a more simplified system. Altough we showed the clear disadvantages of the methodology of using pairs of simulations for the calculation of contributions of emissions to the concentration of a species, the arguments above justify its further use. However, we recommend to calculate the errors $\epsilon_{\alpha}$ and $\epsilon_{\beta}$ and to take a correction of the results by the factor $\frac{1}{1-\epsilon_{\beta}}$ into account.

Note again that the investigation of future policy impact is totally unaffected by this consideration (see Sect. 1).

\section{Conclusions}

Two methodologies have been compared, which calculate the contribution of an emission category, e.g. road traffic, industry, biomass burning, etc. on the atmospheric concentration of gases, which depend on the concentration of the emitted species via chemical reactions. The first method is an accounting system, following the relevant reaction pathways, called tagging method. The second is the calculation of the contributions via two simulations, where one includes a change of $\alpha$ in the regarded emission category. Conceptually, the contribution is calculated by multiplying the total emission of the respective category with the sensitivity of the system, which is the derivative with respect to the emissions. To calculate the derivative most accuratley a small value of $\alpha$ is recommendable.

Both methods were previously used in global modelling studies, though the tagging method was only applied in a more simplified manner, e.g. not considering the combined 
effect of different emitted species of one category on the regarded species, e.g. the combined effect of road traffic $\mathrm{NO}_{\mathrm{x}}$ and non-methane hydrocarbon emissions on ozone.

Note, that the sensitivity method, based on its concept, is inappropriate for source attribution, but well suited to address impacts of e.g. future emission policies. However, since neither a full tagging of the modelled chemistry schemes, nor a tagging system with interrelationships between $\mathrm{NO}_{\mathrm{x}}$ and VOC emissions has been implemented in models, we see the need for a further use of the sensitivity method, though inapproriate for source attribution. In order to assess some principle short-comings, we have introduced two error calculations (see below), which we recommend.

Two very simplified chemical schemes, which represent the main characteristics of atmospheric ozone chemistry, e.g. $\mathrm{NO}_{\mathrm{x}}$-limited and VOC limited regions, ozone titration effects, were developed to test the methodologies. They are based on 3 species, two emitted pre-cursors and one chemically controlled species, which can be regarded as $\mathrm{NO}_{\mathrm{x}}$, VOCs and ozone.

Since the two chemical schemes are simple enough, we were able to provide analytical solutions for the steady-state concentrations and the contributions based on either method. And hence the calculated contributions via the method based on pairs of simulations can be tested against the exact contributions calculated via the tagging methodology.

These theoretical examples show large errors in the calculated contributions for the method, which is based on pairs of simulations, which can easily be a factor of 2 . The error is reduced in many cases when the emission change $(\alpha)$ is small, e.g. $5 \%$. However, a strict convergence is only found for the first chemical system, which is characterised by a more linear system, compared to the second chemical system.

For global scale chemical simulations, these results are likely to be valid for specific local conditions, e.g. in the boundary layer of urban areas. However, on the global or hemispheric scale a more linear behaviour of the chemistry is often found, e.g. for air traffic emissions (Grewe et al., 1999) or lightning emissions (Wild, 2007).

We have provided two error characteristics, which can be calculated quite easily in global simulations and which provide an estimate on how accurate the calculation of the attribution of ozone contributions to individual emission categories are. The first error $\epsilon_{\alpha}$ gives an indication whether the value $\alpha$ has been chosen appropriately, which implies that the contribution from different emissions categories can be intercompared, though the absolute value might have a bias. The second error $\epsilon_{\beta}$ describes this bias. Earlier studies showed that values between $-40 \%$ to $5 \%$ are likely.

\section{Appendix A}

\section{Convergence of the tagging method}

For two given solutions $\left(X_{i}^{1}, Y_{i}^{1}, Z_{i}^{1}\right)$ and $\left(X_{i}^{2}, Y_{i}^{2}, Z_{i}^{2}\right)$ of (11)-(13) with two different initial conditions the solutions exponentially converge to zero: We consider the difference $\Delta X=X_{i}^{1}-X_{i}^{2}$, which fullfills the following differential equation:

$$
\begin{aligned}
\frac{d}{d t} \Delta X & =\left(E_{\mathrm{X}, i}-\tau_{\mathrm{X}}^{-1} X_{i}^{1}\right)-\left(E_{\mathrm{X}, i}-\tau_{\mathrm{X}}^{-1} X_{i}^{2}\right) \\
& =-\tau_{\mathrm{X}}^{-1} \Delta X,
\end{aligned}
$$

and hence converges to zero for any initial difference $\Delta X$. (Similar with the difference $Y_{i}^{2}-Y_{i}^{1}$ ). Therefore we can write $X_{i}^{2}=X_{i}^{1}+\epsilon_{\mathrm{X}}$ and $Y_{i}^{2}=Y_{i}^{1}+\epsilon_{\mathrm{Y}}$ with $\epsilon_{\mathrm{X}}, \epsilon_{\mathrm{Y}}$ converging to zero. The difference $\Delta Z=Z_{i}^{2}-Z_{i}^{1}$ fullfills the equation

$$
\begin{aligned}
\frac{d}{d t} \Delta Z= & -P_{\mathrm{XY}}\left(\epsilon_{\mathrm{X}} Y+\epsilon_{\mathrm{Y}} X\right) \\
& -\left(D_{\mathrm{X}} \epsilon_{\mathrm{X}}+D_{\mathrm{Y}} \epsilon_{\mathrm{Y}}\right) Z \\
& -\left(D_{\mathrm{X}} X+D_{\mathrm{Y}} Y\right) \Delta Z \\
= & Q-\left(D_{\mathrm{X}} X+D_{\mathrm{Y}} Y\right) \Delta Z,
\end{aligned}
$$

where $Q$ converges to zero and therefore also $\Delta Z$.

\section{Appendix B}

\section{General tagging formula}

In atmospheric chemical reaction systems, most reactions are three body reactions at most. Here we consider more generally an arbitrary reaction with $m$ species and $n$ emission categories. (Note that we have used $(m=) 2$ and $(m=) 3$ body reactions in our analysis.) We denote the species $X^{k}$ and the tagged species $\mathrm{X}_{j}^{k}$ for $k \in\{1, \ldots, n\}=N$ and $j \in\{1, \ldots, m\}=M$ and hence the reactions

$$
\begin{aligned}
\mathrm{X}^{1}+\mathrm{X}^{2}+\ldots+\mathrm{X}^{m} & \longrightarrow \text { Products } \\
\mathrm{X}_{j_{1}}^{1}+\mathrm{X}_{j_{2}}^{2}+\ldots+\mathrm{X}_{j_{m}}^{m} & \longrightarrow{\text { Prod. } j_{1}+\ldots+{\text { Prod. } j_{m}},} \text {. }
\end{aligned}
$$

with $j_{1}, \ldots, j_{m} \in M$. Note that in our chemical reaction systems (Sect. 2) $\mathrm{X}, \mathrm{Y}, \mathrm{Z}$ would equal $\mathrm{X}^{1}, \mathrm{X}^{2}, \mathrm{X}^{3}$ in this notation; and the tagged species $\mathrm{X}_{j}, \mathrm{Y}_{j}$, and $\mathrm{Z}_{j}$ equal $\mathrm{X}_{j}^{1}, \mathrm{X}_{j}^{2}$, $\mathrm{X}_{j}^{3}$.

Both the loss of a species $X_{j}^{k}$ and the Products ${ }_{j}$ have contributions from all involved tagged species. Since $m$ species are involved each contributes with the fraction $\frac{1}{m}$. The reaction rate of Reaction (R14) is the rate coefficient times the product $\prod X^{k}$, which equals

$\prod_{k=1}^{m} \sum_{j=1}^{n} X_{j}^{k}=\sum_{j=1}^{n} Q_{j}$

where $Q_{j}$ is the contribution from emission category $j$ to this 
reaction. To obtain the reaction rate for either a loss of $X_{j}^{k}$ or the Products ${ }_{j}$ this product has to be resorted as indicated, which gives

$$
\begin{aligned}
Q_{j} & =\sum_{s=0}^{m-1} \frac{m-s}{m} \sum_{\mathcal{G} \in \mathbb{G}_{s}^{n}} \sum_{g \in \mathcal{G}} \sum_{\mathcal{L} \in \mathbb{L}_{s}^{m}} \prod_{l \in \mathcal{L}} \mathrm{X}_{g}^{l} \prod_{k \in M \backslash \mathcal{L}} \mathrm{X}_{j}^{k}, \\
\mathbb{G}_{s}^{n} & =\left\{\mathcal{G}=\left\{g_{1}, \ldots, g_{s}\right\} \mid g_{1} \leq \ldots \leq g_{s} \in N \backslash\{j\}\right\} \text { and } \\
\mathbb{L}_{s}^{m} & =\left\{\mathcal{L}=\left\{l_{1}, \ldots, l_{s}\right\} \mid l_{1}<\ldots<l_{s} \in M\right\} .
\end{aligned}
$$

The index $s$ indicates how many elements in the product $X_{j_{1}}^{1} X_{j_{2}}^{2} \ldots X_{j_{m}}^{m}$ are not from category $j$ or in other words $s=\left|\left\{j_{i} \mid i \in M, j_{i} \neq j\right\}\right|$. And hence $m-s$ species in the product are from category $j$, which gives $m-s$ times the individual contribution $1 / m$, which is the factor in (B2).

Acknowledgements. This work was supported by the European Union FP6 Integrated Project QUANTIFY (http://www.pa.op.dlr.de/quantify/) A part of the work was performed during a stay at the National Center of Atmospheric Research, Boulder, CO and VG likes to thank G. Pfister, B. Randel, J. F. Lamarque for fruitful discussions and their hospitality. Eleni Tsati has been sponsored by the Helmholtz-PhD-Program of the Helmholtz-Forschungsnetzwerk "Integriertes Erdbeobachtungssystem" (HGF-EOS). Peter Hoor was also supported through the EU-project POMODORO.

Edited by: A. Stenke

\section{References}

Ehhalt, D. H. and Rohrer, F.: The impact of commercial aircraft on tropospheric ozone, Proc. of the 7th BOC Priestly conference, Lewisburg, Pennsylvania, USA, 1994.

Fiore, A. M., Dentener, F. J., Wild, O., et al.: Multimodel estimates of intercontinental source-receptor relationships for ozone pollution, J. Geophys. Res., 114, D04301, doi:10.1029/2008JD010816, 2009.

Fuglestvedt, J. S., Berntsen, T., Myhre, G., Rypdal, K., and Skeie, R. B.: Climate forcing from the transport sectors, P. Natl. Acad. Sci. USA, 105(2), 454-458, 2008.

Grewe, V., Dameris, M., Hein, R., Köhler, I., and Sausen, R.: Impact of future subsonic aircraft $\mathrm{NO}_{\mathrm{x}}$ emissions on the atmospheric composition, Geophys. Res. Lett., 26, 47-50, 1999.

Grewe, V.: Technical Note: A diagnostic for ozone contributions of various $\mathrm{NO}_{\mathrm{x}}$ emissions in multi-decadal chemistryclimate model simulations, Atmos. Chem. Phys., 4, 729-736, doi:10.5194/acp-4-729-2004, 2004.

Grewe, V.: Impact of climate variability on tropospheric ozone, Sci. Total Environ., 374, 167-81, 2007.
Grewe, V.: Impact of lightning on air chemistry and climate, in: Lightning: Principles, Instruments and Applications, edited by: Betz, H. D., Schumann, U., and Laroche, P., Springer Science+Business Media B.V., doi:10.1007/978-1-4020-9079-0_1, 2009.

Gromov, S., Jöckel, P., Sander, R., and Brenninkmeijer, C. A. M.: A kinetic chemistry tagging technique and its application to modelling the stable isotopic composition of atmospheric trace gases, Geosci. Model Dev., 3, 337-364, doi:10.5194/gmd-3-337-2010, 2010.

Hoor, P., Borken-Kleefeld, J., Caro, D., Dessens, O., Endresen, O., Gauss, M., Grewe, V., Hauglustaine, D., Isaksen, I. S. A., Jöckel, P., Lelieveld, J., Myhre, G., Meijer, E., Olivie, D., Prather, M., Schnadt Poberaj, C., Shine, K. P., Staehelin, J., Tang, Q., van Aardenne, J., van Velthoven, P., and Sausen, R.: The impact of traffic emissions on atmospheric ozone and $\mathrm{OH}$ : results from QUANTIFY, Atmos. Chem. Phys., 9, 3113-3136, doi:10.5194/acp-9-3113-2009, 2009.

Horowitz, L. and Jacob, D.: Global impact of fossil fuel combustion on atmospheric $\mathrm{NO}_{\mathrm{x}}$, J. Geophys. Res., 104(D19), 23823-23840, doi:10.1029/1999JD900205, 1999.

Lee, D. S., Pitari, G., Grewe, V., Gierens, K., Penner, J. E., Petzold, A., Prather, M. J., Schumann, U., Bais, A., Berntsen, T., Iachetti,D., Lim, L. L., and Sausen, R.: Transport impacts on atmosphere and climate: Aviation, Atmos. Environ., 44, 46784734, doi: 10.1016/j.atmosenv.2009.06.005, 2010.

Pfister, G. G., Emmons, L. K., Hess, P. G., Lamarque, J.-F., Orlando, J. J., Walters, S., Guenther, A., Palmer, P. I., and Lawrence, P. J.: Contribution of isoprene to chemical budgets: Amodel tracer study with the NCAR CTM MOZART-4, J. Geophys. Res., 113, D05308, doi:10.1029/2007JD008948, 2008.

Seinfeld, J. H. and Pandis, S. N.: Atmospheric Chemistry and Physics, Second edition, John Wiley \& Sons, Inc., Hoboken, New Jersey, USA, 2006.

TRADEOFF - Final report of the EU-project TRADEOFF: Aircraft emissions: Contribution of various climate compounds to changes in composition and radiative forcing - tradeoff to reduce atmospheric impact, 2003.

Wang, Z. S., Chien, C.-J., and Tonnesen, G. S.: Development of a tagged species source apportionment algorithm to characterize three-dimensional transport and transformation of precursors and secondary pollutants, J. Geophys. Res., 114, D21206, doi:10.1029/2008JD010846, 2009.

Wild, O.: Modelling the global tropospheric ozone budget: exploring the variability in current models, Atmos. Chem. Phys., 7, 2643-2660, doi:10.5194/acp-7-2643-2007, 2007.

Wu, S., Duncan, B. N., Jacob, D. J., Fiore, A. M., and Wild, O.: Chemical nonlinearities in relating intercontinental ozone pollution to anthropogenic emissions, Geophys. Res. Lett., 36, L05806, doi:10.1029/2008GL036607, 2009.

Zimmermann, P.: Ozone in the troposphere over Europe: a source segregated analysis from a global point of view, in: Proccedings of EUROTRAC Symposium'98, edited by: Borrell, P. M. and Borell, P., WITPress, Southampton, 1999. 